\title{
A Rapid Microwave Induced Synthesis of Isonicotinamide Derivatives and their Antifungal Activity
}

\author{
Valentina Bušić, ${ }^{1}$ Karolina Vrandečić, ${ }^{2}$ Tamara Siber, ${ }^{2}$ Sunčica Roca, ${ }^{3}$ Dražen Vikić-Topić,, 3 , Dajana Gašo-Sokač ${ }^{1, *}$
}

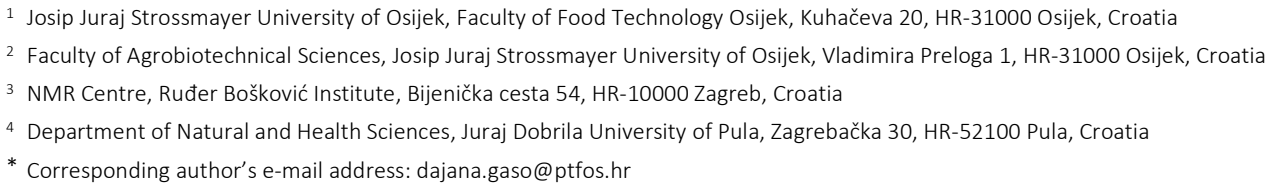

RECEIVED: May 10, $2019 \star$ REVISED: July 3, $2019 \star$ ACCEPTED: July 5, 2019

Abstract: Ten novel isonicotinamide derivatives were prepared by quaternization reactions of isonicotinamide with methyl iodide and nine differently substituted 2-bromoacetophenones under rapid microwave irradiation of 10 minutes. The microwave preparations were significantly faster and with yields higher up to 8 times, than the preparations by conventional method. The structures of synthesized molecules were determined by one- and two-dimensional NMR and IR spectroscopy, mass spectrometry and elemental analysis. Antifungal activity of all compounds was tested in two different concentrations (10 and $100 \mu \mathrm{g} \mathrm{mL}^{-1}$ ) against Fusarium oxysporum, Fusarium culmorum, Macrophomina phaseolina and Sclerotinia sclerotiorum in vitro. From the antifungal assay it can be seen that the most prepared compounds have moderate to weak activity against M. phaseolina and F. culmorum. A very high inhibitory rate was observed against S. sclerotiorum, $62-87.5 \%$ in concentration of $10 \mu \mathrm{g} \mathrm{mL}-1$ and $83.7-93.2 \%$ in concentration of $100 \mu \mathrm{g} \mathrm{mL}^{-1}$.

Keywords: rapid microwave synthesis, quaternization, isonicotinamide derivatives, NMR, antifungal activity.

\section{INTRODUCTION}

$\mathbf{T}$ HE chemistry of carboxamide derivatives with various structural moieties have attracted much attention owing to their different biological properties: antimicrobial, ${ }^{[1-6]}$ antifungal, ${ }^{[7-10]}$ anti-inflammatory, ${ }^{[11]}$

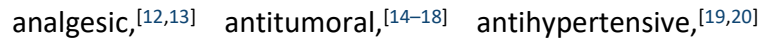
anticonvulsant, ${ }^{[21,22]}$ and antiviral ${ }^{[23]}$ in the recent two decades. More than 255 of known drugs have a carboxamide group in its structure. The bioassay results have shown that some of them exhibited excellent antifungal activities. ${ }^{[23-28]}$ It has been proven that pyridine carboxamide group display fungicidal activity by disrupting the mitochondrial tricarboxylic acid cycle (TCA) through inhibition of the succinate dehydrogenase (SDH) enzyme, also called succinate ubiquinone oxidoreductase (SQR). ${ }^{[29]}$

The most of fungicides are heterocyclic toxic substances which selectively destroy fungal phytopatho- gens (Figure 1). Boscalide, for example, is a pyridine carboxamide mainly used against Botrytis cinerea, Alternaria solani and Sclerotinia sclerotiorum on the fruits and vegetables. ${ }^{[30]}$ Furametpyr and penthiopyrad contain a pyrazole ring in its structure. Furametpyr is widely used to control sheath blight in rice. Penthiopyrad controls rust, Rhizoctonia and other diseases on cereals. ${ }^{[31]}$ Carbendazim, thiabendazole and benomyl have benzimidazole moiety. Carbendazim is used to control plant diseases in cereals and fruits. Studies have found that high doses of carbendazim cause infertility and destroy the testicles of laboratory animals. ${ }^{[32]}$ Benomyl is forbidden for use in EU because of its harmful effects on human development/fertility. Thiabendazole is a post-harvest fungicide mainly used to control a wide range of diseases for fruit and other crops (Aspergillus, Botrytis, Cladosporium, Fusarium spp.). Azoxystrobin, cyproconazole and mancozeb belong to strobilurin and dithiocarbamates groups of compounds.

(cc) $\mathbf{B Y}$ This work is licensed under a Creative Commons Attribution 4.0 International License. 
<smiles>O=C(Nc1ccccc1-c1ccc(Cl)cc1)c1cccnc1Cl</smiles>

Boscalide<smiles>COC(=O)Nc1nc2ccccc2[nH]1</smiles>

Carbendazim<smiles>Cc1nn(C)c(Cl)c1C(=O)Nc1cccc2c1C(C)OC2(C)C</smiles>

Furametpyr<smiles>c1ccc2[nH]c(-c3cscn3)nc2c1</smiles>

Thiabendazole<smiles></smiles>

Penthiopyrad<smiles>CCCCNC(=O)n1c(NC(=O)OC)nc2ccccc21</smiles>

Benomyl<smiles>CO/C=C(/C(=O)OC)c1ccccc1Oc1cc(Oc2ccccc2C#N)ncn1</smiles>

Azoxystrobin<smiles>CC(C1CC1)C(O)(Cn1cncn1)c1ccc(Cl)cc1</smiles>

Cyproconazole<smiles>CCCNC(=O)SN1CCCCC1</smiles>

Mancozeb

Figure 1. Commercial fungicides.

Azoxystrobin possesses the broadest spectrum of activity of all known antifungals. It is the only counteragent that has the ability to protect plants against the four big groups of fungal and fungal-like diseases: Ascomycota, Deuteromycota, Basidiomycota and Oomycota. Cyproconazole is used on cereal crops, coffee, sugar beet, fruit trees and grapes, on sod farms and golf courses and on wood as a preservative. The European Community classifies cyproconazole into carcinogen and reproduction risk category, as harmful if swallowed, and dangerous for the environment (very toxic to aquatic organisms). Mancozeb controls many fungal diseases in a wide range of field crops, fruits, nuts, vegetables, and ornamentals. ${ }^{[31]}$

In a continuation of our previous work ${ }^{[33]}$ in which we have confirmed the antifungal activity of nicotinamide derivatives obtained by quaternization of nicotinamide and substituted 2-bromoacetophenones, we have investigated here the effects of newly prepared isonicotinamide derivatives in vitro against four fungal pathogens (Fusarium oxysporum, Fusarium culmorum, Macrophomina phaseolina and Sclerotinia sclerotiorum).
S. sclerotiorum is a polyphagus which prefers colder climatic areas with high relative humidity in the air. It infects more than 400 plant species from different plant families causes a disease called white mold. ${ }^{[34]}$ The most important hosts of this species are sunflower, oil seed rape, tobacco, soybeans, cabbage and cucumbers. S. sclerotiorum considered the most important disease causing stalk rot of soybean, and can significantly reduce the quality and quantity of yield and increase economic damage. In Europe, Argentina and the United States, due to the occurrence of the white mold, average annual yield reduction of $10-20 \%$ was recorded, and in the years with epidemic proportions damages can be up to $100 \%$. After the occurrence of Sclerotinia in the United States in 2009, the damage was estimated at 560 million USD. ${ }^{[35]} M$. phaseolina is a facultative parasite that causes premature drying of plants. About 500 cultivated and wild plants (sunflower, soybean, sugar beet, alfalfa, maize, tobacco, sweet potatoes) are hosts for this fungus. The seedling, leaf blight, dry rot, charcoal rot disease are different names associated with this fungus since infection can occur in all developmental 


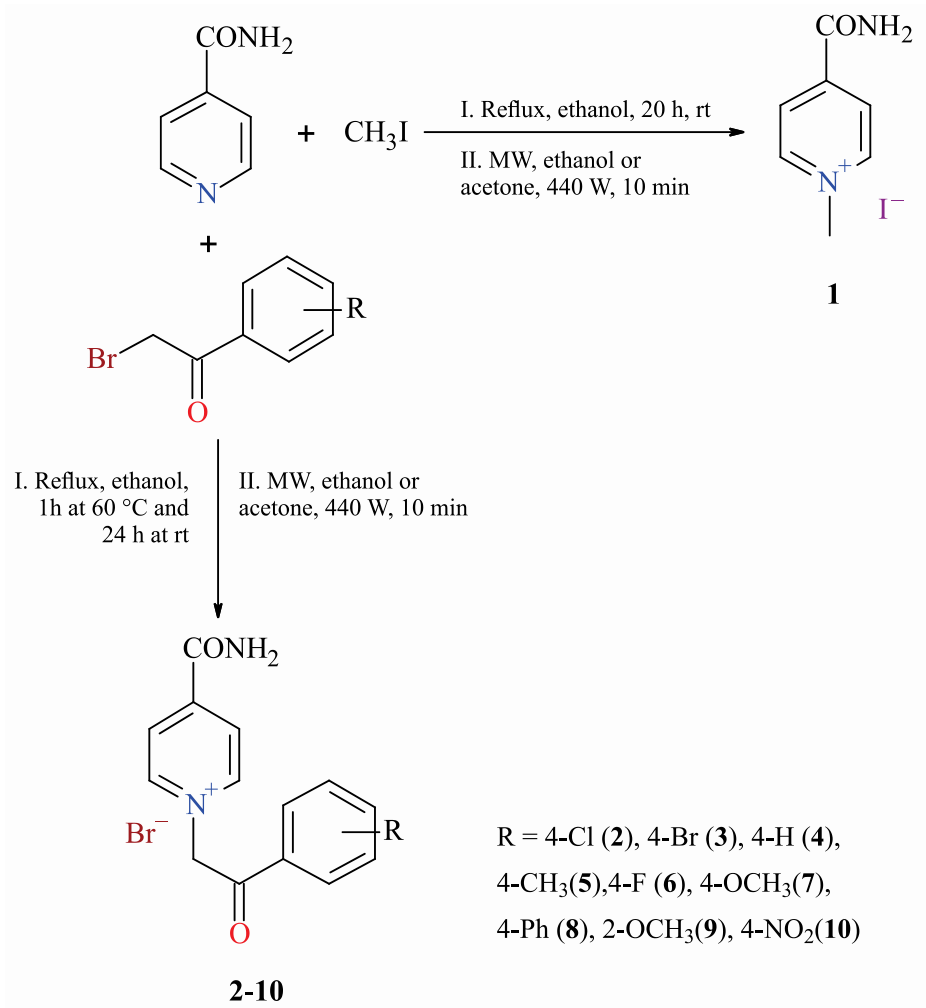

Scheme 1. Synthetic routes of 4-carbamoyl-1-methylpyridinium iodide (1) and to isonicotinamide derivatives with substituted 2-bromoacetophenones (2-10).

stages of the host. In the Pannonian region, $20-25 \%$ lower yield of sunflower and soya have been reported. ${ }^{[35]}$ In Venezuela in 2004, the yield was less than $36-79.2 \%$, ${ }^{[36]}$ and in Russia even up to $64 \% .{ }^{[37]}$ Fusarium genus are one of the most important economic genera of fungi which attack a large number of cultivated plants (wheat, barley, maize, potatoes, tomatoes, cucumbers) and cause different types of diseases (spleen germs, rotting roots and tubers, stalk rot, head blight, ear rot). According to Ćosić and Jurković, during the four years of research one of the most pathogenic species for wheat seedlings was F. culmorum. ${ }^{[38]}$ In this study, we tested whether the derivatives with the quaternary nitrogen atom and the phenacyl unit are effective fungicides comparing to some commercial agricultural ones. Since our new fungicides were originated from vitamin B3 derivatives, we assume that they are nontoxic to humans, animals or plants and that they are less environmentally toxic (Scheme 1). Commercial fungicides from the strobilurin and dithiocarbamates group that have been approved for a specific culture of fungi in Croatia (azoxystrobin, cyproconazole and mancozeb in Figure 1) have been used as standard. Microwave technique for preparation of quaternary isonicotinamide salts was performed in two different solvents, ethanol and acetone.

\section{EXPERIMENTAL}

\section{MW Synthesis and Analysis of Isonicotinamide Salts}

Microwave-assisted synthesis was performed in a controllable single-mode microwave reactor with a magnetic stirring, temperature and power controls (220 V/50-60 Hz, 2.4 kW), Microwave Synthesis Labstation Start $\mathrm{S}$ (Milestone, Shelton, Connecticut, USA). Solvents and reagents were subjected from Merck (Darmstadt, Germany) and Eurisotop (Saint-Aubine, France) and used without further purification.

For elemental analysis PerkinElmer $2400 \mathrm{CHNS} / \mathrm{O}$ Series II System was used (PerkinElmer Inc., Waltham, MA 02451, USA). IR spectra were collecting on Cary 630 FTIR, Agilent Technologies (Santa Clara, CA, USA). Spectroscopic information on molecular ions has also been obtained through the API 2000 LC-MS/MS (Applied Biosystems, USA) where the molecular ions of synthesized compounds in q1 ms scan mode were obtained. Melting points were determined using the electrothermal melting point apparatus SMP $^{3}$ (Mettler Toledo, Croatia).

${ }^{1} \mathrm{H}$ NMR spectra were recorded at $600.133 \mathrm{MHz}$ and ${ }^{13} \mathrm{C}$ NMR spectra at $150.917 \mathrm{MHz}$ using a Bruker AV600 
spectrometer (Rheinstetten, Germany) at Ruđer Bošković Institute. Chemical shifts were referenced to the residual solvent peak (DMSO- $d_{6},{ }^{1} \mathrm{H}$ at $2.50 \mathrm{ppm},{ }^{13} \mathrm{C}$ at $39.51 \mathrm{ppm}$ ). Two-dimensional NMR experiments, ${ }^{1} \mathrm{H}-{ }^{1} \mathrm{H}$ COSY and ${ }^{1} \mathrm{H}-{ }^{13} \mathrm{C}$ $\mathrm{HMBC}$, were performed in order to assist in signal assignment. All spectra were recorded at $298 \mathrm{~K}$ in $5 \mathrm{~mm}$ NMR tubes.

\section{Synthesis of 4-carbamoyl-1-methylpyridinium iodide (1)}

I. Conventional Method: In isonicotinamide $(0.244 \mathrm{~g}, 2$ $\mathrm{mmol}$ ) dissolved in $10 \mathrm{~mL}$ of abs ethanol, was added methyl iodide $(0.187 \mathrm{~mL}, 3 \mathrm{mmol})$, which was also previously dissolved in $10 \mathrm{~mL}$ of abs ethanol. The reaction mixture was refluxed for 20 hours until the product was visible on thin layer chromatography (TLC). Slow cooling of the reaction mixture promoted formation of yellow crystals. Crude product were washed with diethyl ether to remove the residual methyl iodide and recrystallized from methanol.

II. MW synthesis in abs. ethanol or acetone: Isonicotinamide ( $0.244 \mathrm{~g}, 2 \mathrm{mmol}$ ) was dissolved in $10 \mathrm{~mL}$ abs ethanol or acetone and methyl iodide $(0.187 \mathrm{~mL}, 3 \mathrm{mmol})$ was added. The reaction mixture was subjected to $\mathrm{MW}$ irradiation (10 minutes at $440 \mathrm{~W}$ ) until the product was visible on TLC. Slow cooling of the reaction mixture promoted formation of yellowish crystals. The crude product was washed with diethyl ether and recrystallized from methanol.

Synthesis of isonicotinamide products with substituted 2bromoacetophenones unit (2-10)

I. Conventional method: Isonicotinamide $(0.244 \mathrm{~g}, 2 \mathrm{mmol})$ and substituted 2-bromoacetophenone $(2 \mathrm{mmol})$ were dissolved in $100 \mathrm{~mL}$ of acetone. The reaction mixture was mixed on magnetic stirrer and heated for 1 hour at $60{ }^{\circ} \mathrm{C}$, and for another 24 hours at room temperature. The solid precipitate was filtered and washed with acetone. The reaction course was followed by TLC with a mixture of chloroform: methanol $(6: 1)$. Recrystallization from appropriate solvent obtained pure product.

II. MW synthesis: To a solution of isonicotinamide $(0.244 \mathrm{~g}$, $2 \mathrm{mmol}$ ) in $10 \mathrm{~mL}$ of solvent (abs. ethanol or acetone), substituted 2-bromoacetophenones $(2 \mathrm{mmol}$ ) were added and the reaction mixture was irradiated at $440 \mathrm{~W}$ for a 10 minutes, and cooled to room temperature to grow more crystals. The crude products were collected by filtration, washed with diethyl ether and acetone and recrystallized from methanol.

\section{4-carbamoyl-1-methylpyridinium iodide (1)}

Yield: conventional method, $341 \mathrm{mg}, \mathrm{MW}$ from ethanol 489 $\mathrm{mg}$, MW from acetone $389 \mathrm{mg}$; m.p. $208-210^{\circ} \mathrm{C}$; IR (KBr) $v_{\max } / \mathrm{cm}^{-1}: 3362.1,3160.8,3011.7,2117.1,1923.3,1662.4$,
1461.1, 1386.6, 1274.7, 1103.3, 715.6; ${ }^{1} \mathrm{H}$ NMR (DMSO- $d_{6}$ ) $\delta /$ ppm: 9.16 (d, 2H, J=6.44 Hz, H-2, H-6), 8.64 (br s, $1 \mathrm{H}$, $\mathrm{NHa}), 8.40(\mathrm{~d}, 2 \mathrm{H}, J=6.44 \mathrm{~Hz}, \mathrm{H}-3, \mathrm{H}-5), 8.24$ (br s, $1 \mathrm{H}, \mathrm{NHb}$ ), $4.40\left(\mathrm{~s}, 3 \mathrm{H}, \mathrm{CH}_{3}\right) ;{ }^{13} \mathrm{C}$ NMR (DMSO- $\left.d_{6}\right) \delta / \mathrm{ppm}: 163.3$ $\left(\mathrm{CONH}_{2}\right), 147.8$ (C-4), 146.4 (C-2, C-6), 125.3 (C-3, C-5),48.0 $\left(\mathrm{CH}_{3}\right) ; \mathrm{MS} \mathrm{m} / \mathrm{z} 137$ [M+] (100); Anal. Calcd. mass fractions of elements, $w / \%$, for $\mathrm{C}_{7} \mathrm{H}_{9} \mathrm{~N}_{2} \mathrm{OI}\left(M_{\mathrm{r}}=263.0\right)$ : $\mathrm{C} 31.96, \mathrm{H}$ 3.07, I 48.24, N 10.56, $06.08 \%$; found: C 31.88, H 3.25, N $10.71 \%$.

4-carbamoyl-1-(2-(4-chlorophenyl)-2-oxoethyl)pyridin-1ium bromide (2)

Yield: conventional method $270 \mathrm{mg}, \mathrm{MW}$ from abs. ethanol $490 \mathrm{mg}$, MW from acetone $420 \mathrm{mg}$; m.p. $271.5-272^{\circ} \mathrm{C}$, IR $(\mathrm{KBr}) v_{\max } / \mathrm{cm}^{-1}: 3997.0,3235.3,3160.8,2892.4,2668.8$, 2109.7, 1923.3, 1685.5, 1662.4, 1341.8, 1237.5, 923, 820; ${ }^{1} \mathrm{H}$ NMR (DMSO- $\left.d_{6}\right) \delta / \mathrm{ppm}: 9.23(\mathrm{~d}, 2 \mathrm{H}, J=6.91 \mathrm{~Hz}, \mathrm{H}-2, \mathrm{H}-$ 6), $8.78(\mathrm{br} \mathrm{s}, 1 \mathrm{H}, \mathrm{NHa}), 8.58(\mathrm{~d}, 2 \mathrm{H}, J=6.91 \mathrm{~Hz}, \mathrm{H}-3, \mathrm{H}-5)$, 8.32 (br s, $1 \mathrm{H}, \mathrm{NHb}), 8.10$ (d, $2 \mathrm{H}, J=8.65 \mathrm{~Hz}, \mathrm{H}-10, \mathrm{H}-14$ ), $7.76(\mathrm{~d}, 2 \mathrm{H}, \mathrm{J}=8.65 \mathrm{~Hz}, \mathrm{H}-11, \mathrm{H}-13), 6.65\left(\mathrm{~s}, 2 \mathrm{H}, \mathrm{H}-7, \mathrm{H}-\mathrm{7}^{\prime}\right)$; ${ }^{13} \mathrm{C}$ NMR (DMSO- $\left.d_{6}\right) \delta /$ ppm: 189.5 (C-8), $163.4\left(\mathrm{CONH}_{2}\right)$, 149.1 (C-4), 147.1 (C-2, C-6), 139.5 (C-12), 132.2 (C-9), 130.1 (C-10, C-14), 129.2 (C-11, C-13), 125.6 (C-3, C-5), 66.1(C-7); MS $m / z 275.20\left[\mathrm{M}^{+}\right](100) ;$ Anal. Calcd. mass fractions of elements, $w / \%$, for $\mathrm{C}_{14} \mathrm{H}_{12} \mathrm{BrClN}_{2} \mathrm{O}_{2}\left(M_{\mathrm{r}}=355.6\right)$ are: $\mathrm{C}$ 47.29, H 3.40, Br 22.47, Cl 9.97, N 7.88, O 9.00; found: C 47.00, H 3.34, N $7.70 \%$.

1-(2-(4-bromophenyl)-2-oxoethyl)-4-carbamoylpyridin-1ium bromide (3)

Yield: conventional method $360 \mathrm{mg}, \mathrm{MW}$ from abs. ethanol $680 \mathrm{mg}$, MW from acetone $615 \mathrm{mg}$; m.p. $286.3-288^{\circ} \mathrm{C}$, IR $(\mathrm{KBr}) v_{\max } / \mathrm{cm}^{-1}: 3369.5,3131.0,3019.1,2892.4,2668.8$, $1662.4,1565.5,1461.1,1230.0,1073.5,994.0,581.5$, 454.7; ${ }^{1} \mathrm{H}$ NMR (DMSO- $d_{6}$ ) $\delta / \mathrm{ppm}: 9.18(\mathrm{~d}, 2 \mathrm{H}, J=6.74 \mathrm{~Hz}$, $\mathrm{H}-2, \mathrm{H}-6), 8.74$ (br s, $1 \mathrm{H}, \mathrm{NHa}), 8.56$ (d, $2 \mathrm{H}, \mathrm{J}=6.74 \mathrm{~Hz}, \mathrm{H}-3$, $\mathrm{H}-5), 8.31$ (br s, 1H, NHb), 8.01 (d, $2 \mathrm{H}, J=8.53 \mathrm{~Hz}, \mathrm{H}-10, \mathrm{H}-$ 14), 7.91 (d, $2 \mathrm{H}, \mathrm{J}=8.53 \mathrm{~Hz}, \mathrm{H}-11, \mathrm{H}-13), 6.58(\mathrm{~s}, 2 \mathrm{H}, \mathrm{H}-7, \mathrm{H}-$ $\left.7^{1}\right) ;{ }^{13} \mathrm{C}$ NMR (DMSO- $\left.d_{6}\right) \delta / \mathrm{ppm}: 189.7$ (C-8), 163.5 $\left(\mathrm{CONH}_{2}\right.$ ), 149.2 (C-4), 147.1 (C-2, C-6), 132.5 (C-9), 132.2 (C11, C-13), 130.1 (C-10, C-14), 128.8 (C-12), 125.6 (C-3, C-5), 66.1 (C-7); MS m / z $321.10\left[\mathrm{M}^{+}\right](100) ;$ Anal. Calcd. mass fractions of elements, $w / \%$, for $\mathrm{C}_{14} \mathrm{H}_{12} \mathrm{Br}_{2} \mathrm{~N}_{2} \mathrm{O}_{2}\left(M_{\mathrm{r}}=400.0\right)$ : C 42.03, H 3.02, Br 39.95, N 7.00, O 8.00; found: C 41.94, H $2.79, \mathrm{~N} 6.80 \%$.

\section{4-carbamoyl-1-(2-oxo-2-phenylethyl)pyridin-1-ium bromide (4)}

Yield: conventional method $205 \mathrm{mg}$, MW from abs. ethanol $578 \mathrm{mg}$, MW from acetone $497 \mathrm{mg}$; m.p. 236.5-238 ${ }^{\circ} \mathrm{C}$; IR (KBr) $v_{\max } / \mathrm{cm}^{-1}: 3339.7,3235.3,3138.4,2892.4,2832.8$, 2117.1, 1915.8, 1677.3, 1297.1, 1617.7, 1452.7, 1207.7, 1148.0, 984.0, 767.8, $\mathrm{cm}^{-1}$; ${ }^{1} \mathrm{H}$ NMR (DMSO- $d_{6}$ ) $\delta / \mathrm{ppm}$ : $9.23(\mathrm{~d}, 2 \mathrm{H}, \mathrm{J}=6.66 \mathrm{~Hz}, \mathrm{H}-2, \mathrm{H}-6), 8.78(\mathrm{br} \mathrm{s}, 1 \mathrm{H}, \mathrm{NHa}), 8.58$ 
(d, $2 \mathrm{H}, J=6.66 \mathrm{~Hz}, \mathrm{H}-3, \mathrm{H}-5), 8.33$ (br s, $1 \mathrm{H}, \mathrm{NHb}$ ), 8.09 (dd, $2 \mathrm{H}, J=8.17,1.26 \mathrm{~Hz}, \mathrm{H}-10, \mathrm{H}-14), 7.80(\mathrm{tt}, 1 \mathrm{H}, J=7.39,1.01$ $\mathrm{Hz}, \mathrm{H}-12), 7.68$ (t, 2H, J= $7.98 \mathrm{~Hz}, \mathrm{H}-11, \mathrm{H}-13), 6.65$ (s, 2H, $\left.\mathrm{H}-7, \mathrm{H}-7^{\prime}\right) ;{ }^{13} \mathrm{C}$ NMR (DMSO- $\left.d_{6}\right) \delta / \mathrm{ppm}: 190.4$ (C-8), 163.5 $\left(\mathrm{CONH}_{2}\right), 149.1$ (C-4), 147.2 (C-2, C-6), 134.7 (C-12), 133.4 (C-9), 129.1 (C-11, C-13), 128.3 (C-10, C-14), 125.6 (C-3, C5), 66.3 (C-7); MS m / z $241\left[\mathrm{M}^{+}\right](100)$; Anal. Calcd. mass fractions of elements, $w / \%$, for $\mathrm{C}_{14} \mathrm{H}_{13} \mathrm{~N}_{2} \mathrm{O}_{2} \mathrm{Br}\left(M_{\mathrm{r}}=321.2\right)$ : C 52.36, H 4.08, N 8.72 O 9.96; found: C 52.40, H 3.78, N $8.74 \%$.

\section{4-carbamoyl-1-(2-oxo-2-(p-tolyl)ethyl)pyridin-1-ium bromide (5)}

Yield: conventional method $140 \mathrm{mg}, \mathrm{MW}$ from abs. ethanol $610 \mathrm{mg}$, MW from acetone $501 \mathrm{mg}$; m.p. 232.5-250.9 ${ }^{\circ} \mathrm{C}$; IR $(\mathrm{KBr}) v_{\max } / \mathrm{cm}^{-1}: 3324.8,3011.7,2780.6,2087.3,1848.8$, 1808.4, 1677.3, 1446.2, 1386.6, 1118.2, 775.3; ${ }^{1} \mathrm{H}$ NMR (DMSO- $d_{6}$ ) $\delta$ / ppm: 9.22 (d, $\left.2 \mathrm{H}, J=6.95 \mathrm{~Hz}, \mathrm{H}-2, \mathrm{H}-6\right), 8.76$ (br s, $1 \mathrm{H}, \mathrm{NHa}$ ), 8.57 (d, 2H, J = $6.93 \mathrm{~Hz}, \mathrm{H}-3, \mathrm{H}-5$ ), 8.30 (br s, $1 \mathrm{H}, \mathrm{NHb}$ ), 7.98 (d, $2 \mathrm{H}, J=8.24 \mathrm{~Hz}, \mathrm{H}-10, \mathrm{H}-14), 7.48$ (d, $2 \mathrm{H}, \mathrm{J}=8.24 \mathrm{~Hz}, \mathrm{H}-11, \mathrm{H}-13), 6.61\left(\mathrm{~s}, 2 \mathrm{H}, \mathrm{H}-7, \mathrm{H}-7^{\prime}\right), 2.44$ (s, $3 \mathrm{H}, \mathrm{CH}_{3}$ ); ${ }^{13} \mathrm{C}$ NMR (DMSO-d 6 ) $\delta /$ ppm: 189.9 (C-8), 163.5 $\left(\mathrm{CONH}_{2}\right), 149.0$ (C-4), 147.1 (C-2, C-6), 145.4 (C-12), 130.9 (C-9), 129.6 (C-11, C-13), 128.3 (C-10, C-14), 125.5 (C-3, C5), 66.1 (C-7), $21.3\left(\mathrm{CH}_{3}\right) ; \mathrm{MS} \mathrm{m} / \mathrm{z} 255\left[\mathrm{M}^{+}\right]$(100); Anal. Calcd. mass fractions of elements, $w / \%$, for $\mathrm{C}_{15} \mathrm{H}_{15} \mathrm{BrN}_{2} \mathrm{O}_{2}$ $\left(M_{\mathrm{r}}=335.2\right): \mathrm{C} 53.75, \mathrm{H} 4.51, \mathrm{Br} 23.84, \mathrm{~N} 8.36, \mathrm{O} 9.55$; found: C 53.39, H 4.56, N $8.37 \%$.

\section{4-carbamoyl-1-(2-(4-fluorophenyl)-2-oxoethyl)pyridin-1- ium bromide (6)}

Yield: conventional method $266 \mathrm{mg}$, MW from abs. ethanol $480 \mathrm{mg}$, MW from acetone $425 \mathrm{mg}$; m.p. $271.5-272{ }^{\circ} \mathrm{C}$; IR $(\mathrm{KBr}) v_{\max } / \mathrm{cm}^{-1}: 3526.1,3436.8,3265.1,3034.1,2079.9$, 1908.4, 1692.2, 1640,0, 1394.0, 1326.9, 1222.6, 1155.5, 991.5, 820.0, 700.7, 462.2; ${ }^{1} \mathrm{H}$ NMR (DMSO- $d_{6}$ ) $\delta / \mathrm{ppm}$ : $9.22(\mathrm{~d}, 2 \mathrm{H}, J=6.54 \mathrm{~Hz}, \mathrm{H}-2, \mathrm{H}-6), 8.77$ (br s, $1 \mathrm{H}, \mathrm{NHa}), 8.57$ (d, $2 \mathrm{H}, J=6.54 \mathrm{~Hz}, \mathrm{H}-3, \mathrm{H}-5), 8.32$ (br s, $1 \mathrm{H}, \mathrm{NHb}), 8.18$ (m, $2 \mathrm{H}, \mathrm{H}-11, \mathrm{H}-13), 7.52(\mathrm{t}, 2 \mathrm{H}, \mathrm{J}=8.89 \mathrm{~Hz}, \mathrm{H}-10, \mathrm{H}-14), 6.63$ $\left(\mathrm{s}, 2 \mathrm{H}, \mathrm{H}-7, \mathrm{H}-7^{\prime}\right) ;{ }^{13} \mathrm{C}$ NMR (DMSO- $\left.d_{6}\right) \delta / \mathrm{ppm}: 189.1$ (C-8), $165.7\left(\mathrm{~d}, J_{\mathrm{CF}}=253.94 \mathrm{~Hz}, \mathrm{C}-12\right), 163.5\left(\mathrm{CONH}_{2}\right), 149.1(\mathrm{C}-4)$, $147.1(C-2, C-6), 131.4$ (d, J $\left.J_{C F}=9.67 \mathrm{~Hz}, C-10\right), 130.3$ (d, $\left.J_{\mathrm{CF}}=2.10 \mathrm{~Hz}, \mathrm{C}-9\right), 125.6$ (C-3, C-5), 116.3 (d, $J_{\mathrm{CF}}=22.28 \mathrm{~Hz}$, C-11), 66.1 (C-7); MS m / z 259 [M+'] (100); Anal. Calcd. mass fractions of elements, $w / \%$, for $\mathrm{C}_{14} \mathrm{H}_{12} \mathrm{BrFN}_{2} \mathrm{O}_{2}\left(M_{\mathrm{r}}=\right.$ 339.2): C 49.58, H3.57, N 8.26, 09.43; found: C 48.54 H3.74, N $8.01 \%$.

\section{4-carbamoyl-1-(2-(4-methoxyphenyl)-2-oxoethyl)pyridin- 1-ium bromide (7) \\ Yield: conventional method $323 \mathrm{mg}$, MW from abs. ethanol $610 \mathrm{mg}$, MW from acetone $552 \mathrm{mg}$; m.p. $258-259^{\circ} \mathrm{C}$; IR $(\mathrm{KBr}) v_{\max } / \mathrm{cm}^{-1}: 3436.6,3295.0,3116.1,3041.5,2318.4$,}

2117.1, 1908.4, 1677.3, 1565.5, 1423.8, 1237.5, 1118.2, 842.4, 767.8, 566.6.; ${ }^{1} \mathrm{H}$ NMR (DMSO- $d_{6}$ ) $\delta /$ ppm: 9.22 (d, $2 \mathrm{H}, J=6.76 \mathrm{~Hz}, \mathrm{H}-2, \mathrm{H}-6), 8.77$ (br s, $1 \mathrm{H}, \mathrm{NHa}), 8.56$ (d, $2 \mathrm{H}$, $J=7.08 \mathrm{~Hz}, \mathrm{H}-3, \mathrm{H}-5), 8.31$ (br s, $1 \mathrm{H}, \mathrm{NHb}), 8.06$ (d, $2 \mathrm{H}$, $J=8.82 \mathrm{~Hz}, \mathrm{H}-10, \mathrm{H}-14), 7.19$ (d, $2 \mathrm{H}, J=8.93 \mathrm{~Hz}, \mathrm{H}-11, \mathrm{H}-$ 13), $6.59\left(\mathrm{~s}, 2 \mathrm{H}, \mathrm{H}-7 / 7^{\prime}\right), 3.91\left(\mathrm{~s}, 3 \mathrm{H}, \mathrm{OCH}_{3}\right) ;{ }^{13} \mathrm{C} \mathrm{NMR}$ (DMSO- $d_{6}$ ) $\delta /$ ppm: 189.9 (C-8), $163.6\left(\mathrm{CONH}_{2}\right), 150.6$ (C12), 149.3 (C-4), 147.2 (C-2, C-6), 138.2 (C-9), 129.8 (C-10, C-14), 125.7 (C-3, C-5), 124.2 (C-11, C-13), 66.6 (C-7), 55.8 $\left(\mathrm{OCH}_{3}\right) ; \mathrm{MS} \mathrm{m} / \mathrm{z} 271\left[\mathrm{M}^{+}\right]$(100); Anal. Calcd. mass fractions of elements, $w / \%$, for $\mathrm{C}_{15} \mathrm{H}_{15} \mathrm{BrN}_{2} \mathrm{O}_{3}\left(M_{\mathrm{r}}=351.2\right)$ : $\mathrm{C} 51.30$, H 4.31, Br 22.75,N 7.98, O 13.67; found: C50.76, H 4.09, N $7.54 \%$.

\section{1-(2-([1,1'-biphenyl]-4-yl)-2-oxoethyl)-4-} carbamoylpyridin-1-ium bromide (8)

Yield: conventional method $201 \mathrm{mg}, \mathrm{MW}$ from abs. ethanol $490 \mathrm{mg}$, MW from acetone $375 \mathrm{mg}$; m.p. $271-273{ }^{\circ} \mathrm{C}, \mathrm{IR}$ (KBr) $v_{\max } / \mathrm{cm}^{-1}: 3257.7,3086.2,2117.1,1893.5,1677.3$, 1461.1, 1334.4, 842.4, 767.8, 700.7, 447.3; ${ }^{1} \mathrm{H}$ NMR (DMSO-d $d_{6}$ ) $\delta$ / ppm: 9.20 (d, $2 \mathrm{H}, J=6.75 \mathrm{~Hz}, \mathrm{H}-2, \mathrm{H}-6$ ), 8.74 (br s, 1H, NHa), $8.56(\mathrm{~d}, 2 \mathrm{H}, J=6.75 \mathrm{~Hz}, \mathrm{H}-3, \mathrm{H}-5), 8.32(\mathrm{br}$ $\mathrm{s}, 1 \mathrm{H}, \mathrm{NHb}), 8.16(\mathrm{~d}, 2 \mathrm{H}, \mathrm{J}=8.44 \mathrm{~Hz}, \mathrm{H}-10, \mathrm{H}-14), 7.83$ (m, $2 \mathrm{H}, \mathrm{H}-16, \mathrm{H}-20), 7.80$ (d, 2H, J=8.44 Hz, H-11, H-13), 7.55 (t, $2 \mathrm{H}, J=7.40 \mathrm{~Hz}, \mathrm{H}-17, \mathrm{H}-19), 7.48$ (tt, $1 \mathrm{H}, J=7.40,1.11$ $\mathrm{Hz}, \mathrm{H}-18$ ); ${ }^{13} \mathrm{C}$ NMR (DMSO- $d_{6}$ ) $\delta / \mathrm{ppm}: 189.9$ (C-8), 163.5 $\left(\mathrm{CONH}_{2}\right.$ ), 149.1 (C-4), 147.2 (C-2, C-6), 145.9 (C-12), 138.4 (C-15), 132.2 (C-9), 129.1 (C-17, C-19), 129.0 (C-10, C-14), 128.7 (C-18), 127.1 (C-16, C-20), 127.0 (C-11, C-13), 125.6 (C-3, C-5), 66.2 (C-7). MS m / z: 317 [M+] (100); Anal. Calcd. mass fractions of elements, $w / \%$, for $\mathrm{C}_{20} \mathrm{H}_{17} \mathrm{BrN}_{2} \mathrm{O}_{2}\left(M_{\mathrm{r}}=\right.$ 397.3): C 60.47, H 4.31, Br 20.11, N 7.05, O 8.05; found: C $59.94, \mathrm{H} 4.14, \mathrm{~N} 6.68 \%$.

\section{4-carbamoyl-1-(2-(2-methoxyphenyl)-2-oxoethyl)pyridin- 1-ium bromide (9)}

Yield: conventional method $122 \mathrm{mg}, \mathrm{MW}$ from abs. ethanol $287 \mathrm{mg}$, MW from acetone $206 \mathrm{mg} ; \mathrm{m} . \mathrm{p} .245-247^{\circ} \mathrm{C}$; IR $(\mathrm{KBr}) v_{\max } / \mathrm{cm}^{-1}: 3339.7,3235.3,3135.3,2952.1,2117.1$, 1886.0, 1684.8, 1595.3, 1461.1, 1386.6, 1289.7, 1207.7, 909.5, 760.4, 678.4, $588.6 \mathrm{~cm}^{-1}$; ${ }^{1} \mathrm{H}$ NMR (DMSO-d $d_{6}$ ) $\delta /$ ppm: 9.19 (d, 2H, J = $6.50 \mathrm{~Hz}, \mathrm{H}-2, \mathrm{H}-6), 8.75$ (br s, $1 \mathrm{H}$, $\mathrm{NHa}), 8.53(\mathrm{~d}, 2 \mathrm{H}, J=6.50 \mathrm{~Hz}, \mathrm{H}-3, \mathrm{H}-5), 8.30$ (s, 1H, NHb), 7.89 (dd, $1 \mathrm{H}, J=7.70,1.75 \mathrm{~Hz}, \mathrm{H}-10$ ), 7.75 (ddd, 1H, $J=7.70,7.35,1.75 \mathrm{~Hz}, \mathrm{H}-12), 7.37(\mathrm{~d}, 1 \mathrm{H}, J=8.40 \mathrm{~Hz}, \mathrm{H}-13)$, $7.16(\mathrm{t}, 1 \mathrm{H}, J=7.35 \mathrm{~Hz}, \mathrm{H}-11), 6.32\left(\mathrm{~s}, 2 \mathrm{H}, \mathrm{H}-7, \mathrm{H}-7^{\prime}\right), 4.06$ (s, $3 \mathrm{H}, \mathrm{OCH}_{3}$ ); ${ }^{13} \mathrm{C}$ NMR (DMSO- $d_{6}$ ) $\delta / \mathrm{ppm}: 189.7$ (C-8), 163.5 (CONH2), 160.0 (C-14), 148.9 (C-4), 147.1 (C-2, C-6), 136.4 (C-12), 130.3 (C-10), 125.3 (C-3, C-5), 122.8 (C-9), 120.9 (C11), 113.1 (C-13), 69.8 (C-7), $56.3\left(\mathrm{CH}_{3}\right) ; \mathrm{MS} \mathrm{m} / \mathrm{z} 271\left[\mathrm{M}^{+}\right]$ (100); Anal. Calcd. mass fractions of elements, $w / \%$, for $\mathrm{C}_{15} \mathrm{H}_{15} \mathrm{BrN}_{2} \mathrm{O}_{3}\left(M_{\mathrm{r}}=351.2\right): \mathrm{C} 51.30, \mathrm{H} 4.31, \mathrm{Br} 22.75, \mathrm{~N} 7.98$, O 13.67; found: C 51.50, H 4.39, N $7.77 \%$. 
<smiles>NC(=O)c1cc[n+](CCCI)cc1</smiles>

11<smiles>NC(=O)c1cc[n+](CCCBr)cc1</smiles>

12
Figure 2. Structure of compounds 11 and 12 .

\section{4-carbamoyl-1-[2-(4-nitrophenyl)-2-oxoethyl]pyridinium} bromide (10)

Yield: conventional method $422 \mathrm{mg}, \mathrm{MW}$ from abs. ethanol $655 \mathrm{mg}$, MW from acetone $572 \mathrm{mg}$; m.p. $269.0-269.5^{\circ} \mathrm{C}$; IR $(\mathrm{KBr}) v_{\max } / \mathrm{cm}^{-1}: 3536.3,3354.6,3235.3,3116.1,2072.4$, 2005.3, 1930.8, 1692.2, 1625.1, 1394.0, 1341.8, 1215.1, 891.5, 849.8, 685.8; ${ }^{1} \mathrm{H}$ NMR (DMSO- $d_{6}$ ) $\delta / \mathrm{ppm}: 9.24$ (d, $2 \mathrm{H}, J=6.72 \mathrm{~Hz}, \mathrm{H}-2, \mathrm{H}-6), 8.79$ (br s, $1 \mathrm{H}, \mathrm{NHa}$ ), 8.60 (d, $2 \mathrm{H}$, $J=6.72 \mathrm{~Hz}, \mathrm{H}-3, \mathrm{H}-5), 8.49$ (d, $2 \mathrm{H}, J=9.16 \mathrm{~Hz}, \mathrm{H}-11, \mathrm{H}-13$ ), 8.34 (br s, $1 \mathrm{H}, \mathrm{NHb}), 8.33$ (d, $2 \mathrm{H}, \mathrm{J}=9.16 \mathrm{~Hz}, \mathrm{H}-10, \mathrm{H}-14$ ), $6.72\left(\mathrm{~s}, 2 \mathrm{H}, \mathrm{H}-7, \mathrm{H}-7^{\prime}\right) ;{ }^{13} \mathrm{C}$ NMR (DMSO-d $\left.d_{6}\right) \delta / \mathrm{ppm}: 189.9$ (C-8), 163.6 (CONH2), 150.6 (C-12), 149.3 (C-4), 147.2 (C-2, C-6), 138.2 (C-9), 129.8 (C-10, C-14), 125.7 (C-3, C-5), 124.2 (C-11, C-13), 66.6 (C-7); MS m/z $286\left[\mathrm{M}^{+}\right]$(100); Anal. Calcd. mass fractions of elements, $w / \%$, for $\mathrm{C}_{13} \mathrm{H}_{11} \mathrm{~N}_{2} \mathrm{O}_{3} \mathrm{Br}$ : C 48.32, H 3.43, N 8.67. Found: C 48.38, H 3.39, N $8.70 \%$. Compounds 11 and 12 (Figure 2) were synthesized and characterized in our previous paper. ${ }^{[39]}$ In this work we tested their antifungal activity for the sake of comparison with ten newly synthesized isonicotinamide derivatives (1-10).

\section{Antifungal Assay}

Antifungal assay was performed on four culture of phytopathogenic fungi (M. phaseolina, S. sclerotiorum, $F$. oxysporum, F. culmorum) provided from the culture collections of Chair for phytopathology, Faculty of Agrobiotechnical Sciences University of Osijek. The fungicidal activity of twelve synthetic compounds (1-12) at concentrations of $10 \mu \mathrm{g} \mathrm{mL}^{-1}$ and $100 \mu \mathrm{g} \mathrm{mL}^{-1}$ were tested. For antifungal assays the method according to Siber et al. was used. ${ }^{[33]}$ Each treatment was performed in four replicates. As a control, untreated potato dextrose agar (PDA) was used. Commercially agricultural fungicide was used as positive control. For F. culmorum, F. oxysporum and S. sclerotiorum the fungicide from the strobilurin group with two active ingredients (azoxystrobin and cyproconazole) was used. For $M$. phaseolina the fungicide from the dithiocarbamates group with mancozeb as an active ingredient was used. The fungicides were mixed with PDA in the recommended concentration. Inhibition rate of the synthetic compounds on the four fungi species was calculated by the antifungal index ( $/ \%)$

$$
I / \%=[(C-S) /(C-0.4)] \times 100
$$

where $C$ represents the diameter of fungal growth on untreated PDA and $S$ represents the diameter of fungal growth on treated PDA with synthetic compounds.

Statistical analysis of data was performed using factorial analysis of variance ANOVA by grouping the data depending on inhibition rate and concentration applied. For estimate statistical significance of differences between synthetic compounds at different concentrations Fisher's LSD test was applied by using SAS 9.2 Statistical Package. ${ }^{[40]}$ Mean values were considered significantly different when $p \leq 0.05$.

\section{RESULTS AND DISCUSSION}

A demanding step of microwave-assisted synthesis is the finding of optimum conditions for a specific reaction, to obtain the desired products in good yields and purities. Optimum conditions for performing the quaternization reaction were ascertained by carrying out reaction of isonicotinamide and substituted 2-bromoacetophenone. The results are summarized in Table 1 and showed that the maximum yield of quaternary salt was obtained by heating the reaction mixture at $440 \mathrm{~W}$ for $10 \mathrm{~min}$. The long heating time ( $\geq 12 \mathrm{~min}$ ) increases impurities production, which makes difficulties in separation of the desired pure solid product.

Conventional synthesis required longer heating time for isonicotinamide and methyl iodide $(\approx 20 \mathrm{~h})$, while in reaction with substituted 2-bromoacetophenones reflux time of 1 hour at $60{ }^{\circ} \mathrm{C}$, plus 24 hours of stirring at room temperature, is required. Microwave-assisted synthesis under controlled conditions has been proved as a valuable method for any application that requires heating of a reaction mixture, since it often significantly reduces

Table 1. Structure of compound (4) and screening of reaction times in the quaternization reaction between isonicotinamide and 2-bromoacetophenone at $440 \mathrm{~W}$ in ethanol<smiles>NC(=O)c1cc[n+](CC(=O)c2ccccc2)cc1</smiles>

\begin{tabular}{cc}
\hline Time $/$ min & Yield / \% \\
\hline 2 & 30 \\
4 & 38 \\
6 & 56 \\
8 & 78 \\
10 & 90 \\
12 & 75 \\
14 & 80 \\
16 & 84 \\
\hline
\end{tabular}


Table 2. Experimental times and yields of products (1-10) obtained by the conventional and microwave-assisted method, in absolute ethanol and acetone

\begin{tabular}{|c|c|c|c|c|c|c|c|c|c|c|}
\hline Compound & 1 & 2 & 3 & 4 & 5 & 6 & 7 & 8 & 9 & 10 \\
\hline \multicolumn{11}{|c|}{ Conventional method (reflux) } \\
\hline Time (Temperature) & $20 \mathrm{~h}(\mathrm{rt})$ & \multicolumn{9}{|c|}{$1 \mathrm{~h}\left(60^{\circ} \mathrm{C}\right)+24 \mathrm{~h}(\mathrm{rt})$} \\
\hline Solvent & $\mathrm{EtOH}$ & \multicolumn{9}{|c|}{ Acetone } \\
\hline Yield (\%) & 65 & 38 & 45 & 32 & 21 & 40 & 46 & 25 & 15 & 58 \\
\hline \multicolumn{11}{|c|}{ Microwave-assisted method } \\
\hline Time & \multicolumn{10}{|c|}{$10 \mathrm{~min}$} \\
\hline Solvent & \multicolumn{10}{|c|}{ Ethanol } \\
\hline Yield (\%) & 93 & 69 & 64 & 90 & 91 & 71 & 86 & 62 & 61 & 89 \\
\hline Time & \multicolumn{10}{|c|}{$10 \mathrm{~min}$} \\
\hline Solvent & \multicolumn{10}{|c|}{ Acetone } \\
\hline Yield (\%) & 74 & 59 & 77 & 77 & 75 & 63 & 79 & 47 & 29 & 72 \\
\hline
\end{tabular}

reaction times from days or hours to minutes or seconds. All ten compounds were rapidly prepared in very short reaction times $(10 \mathrm{~min})$. The main differences between conventional and microwave synthesis in reaction time and yields are shown in Table 2.

The highest yield (65\%) in the conventional method was obtained in the reaction of isonicotinamide and methyl iodide (1). Among the investigated substituted 2-bromoacetophenones, the highest yield (58\%) was achieved with 2-bromo-4-nitroacetophenone (10). The lowest yield (15\%) was found for compound 9. Other compounds were synthesized in low to moderate yields ( 25 to $46 \%$ ). The nucleophilic substitution of halogen in the $\alpha$-haloketones is considerably easy because of the greater electrophilicity of the carbon adjacent to the acceptor carbonyl group. In isonicotinamide the substituent decreases the nucleophilicity of nitrogen due to its negative inductive effect. Consequently, the presence of a highly acceptor carbonyl group in the substrate, as in the case of phenacyl bromide, leads to redistribution of electron density in the molecule, and the weakening of $\mathrm{C}-\mathrm{Br}$ bond, which resulted in easier removal of the leaving group.

The highest yields by conventional and $\mathrm{MW}$ syntheses were achieved for compound $\mathbf{1}$ where iodine is a leaving group. The halogens in our investigation were bromine and iodine. Halogens have electronegativities significantly greater than carbon. Hence, this functional group is polarized so that the carbon is electrophilic and the halogen is nucleophilic. Two characteristics other than electronegativity also have an important influence on the chemical behavior of these compounds. The first is covalent bond strength. The strongest of the carbon-halogen covalent bonds is that to bromine, so the covalent bond is chemically and thermodynamically quite stable. The carbon-iodine covalent bond is slightly weaker than a carbon-bromine bond, so iodides are better leaving groups in nucleophilic substitution than bromides, which agrees with our yields in reaction with methyl iodide.

As a result of microwave-assisted synthesis, it was observed that the reaction of quaternization was completed in a short time of $10 \mathrm{~min}$ with higher yields compared to the conventional method ca. $20 \mathrm{~h}$ for methyl iodide and one day for substituted 2-bromoacetophenones. Generally, all obtained yields in microwave synthesis are higher in ethanol than in acetone. A solvent with a high $\tan \delta(\tan \delta>0.5)$, like ethanol, is required for rapid heating in the microwave field. Acetone is classified as low microwave absorbing solvent ( $\tan \delta<0.1$ ). The solvents with greater value of $\tan \delta$ more effectively converts microwave energy to thermal energy. ${ }^{[41]}$ The highest yields in microwave-assisted synthesis in ethanol was achieved for compound 5 (91 \%) and in acetone for compound 7 (79\%), while the lowest yields were was obtained for compound 9, in ethanol $61 \%$ and in acetone $29 \%$. The lowest yield for $\mathbf{9}$, in both by conventional and $\mathrm{MW}$ synthesis corresponds with steric hindrance of $-\mathrm{OCH}_{3}$ group in ortho- position of 2-bromoacetophenone which reduces the efficiency of quaternization.

The chemical structures of investigated compounds were determined on the basis of spectral data analysis of $\mathrm{IR},{ }^{1} \mathrm{H}$ and ${ }^{13} \mathrm{C}$ NMR spectra in solution, elemental analysis and mass spectral analysis. All compounds show the absorption bands for $v \mathrm{~N}-\mathrm{H}, v \mathrm{NH}_{2}, v \mathrm{NH}_{2}, v \mathrm{C}=\mathrm{O}$ and $\delta \mathrm{N}-\mathrm{C}-\mathrm{C}$ groups at $3400,3350,3180,1690-1650$ and $1620 \mathrm{~cm}^{-1}$. The absorption bands of aromatic rings, $v C-C A r 1650,1580$, $1400 \mathrm{~cm}^{-1}$ and $v C-H A r 3030,3010 \mathrm{~cm}^{-1}$, were also observed.

Prepared compounds were soluble in DMSO- $d_{6}$ at room temperature. The number of signals and their chemical shifts in ${ }^{1} \mathrm{H}$ and ${ }^{13} \mathrm{C}$ NMR spectra as well as 

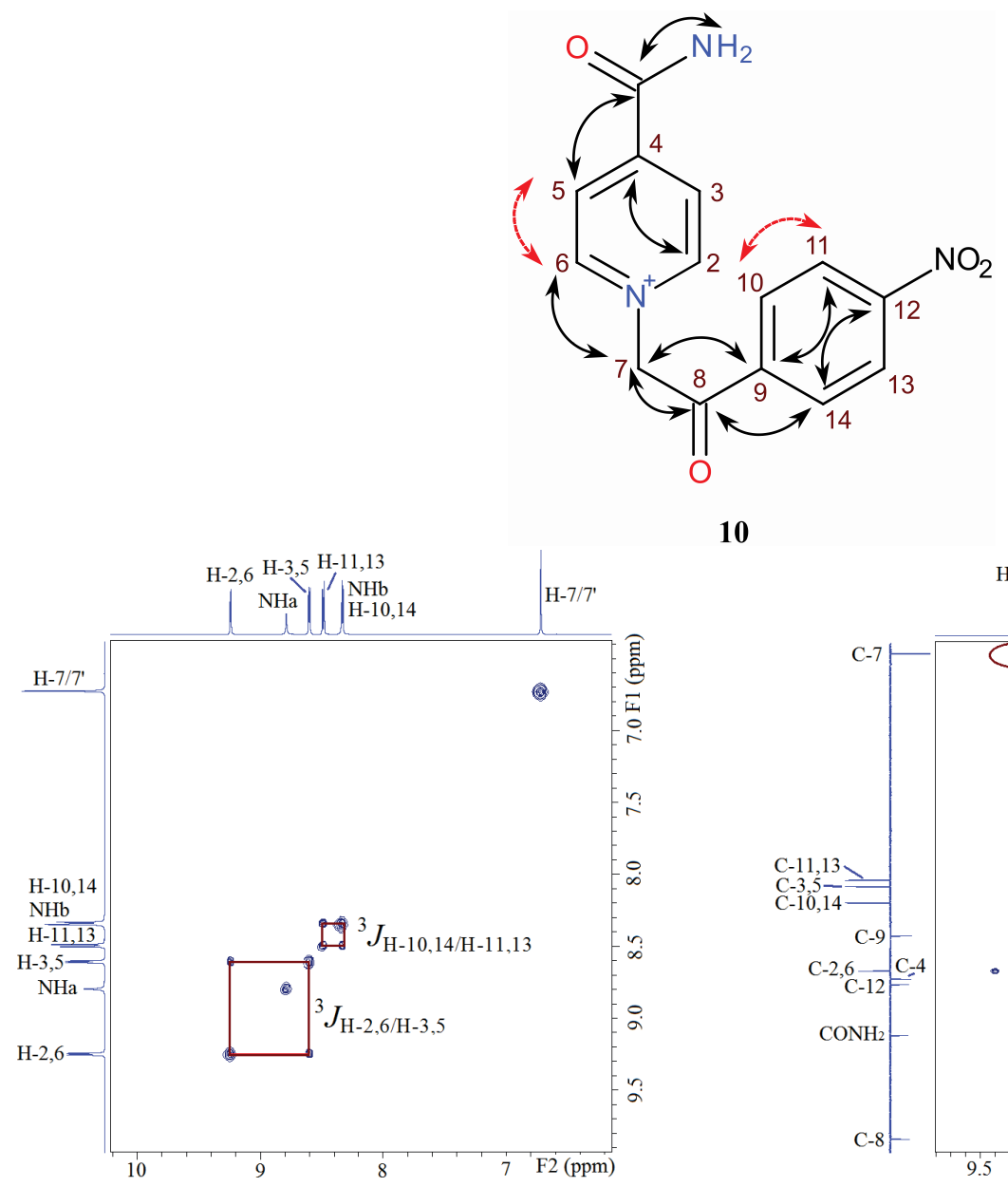

10

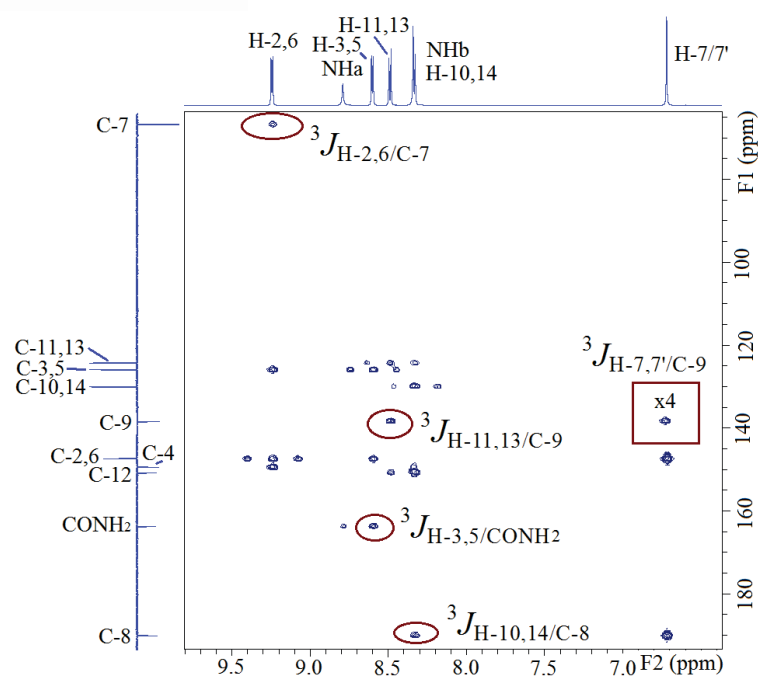

Figure 3. Structure of 10 with the atom-numbering scheme and selected couplings obtained in $(\mathrm{A}){ }^{1} \mathrm{H}-{ }^{1} \mathrm{H}$ COSY (dashed arrows) and (B) ${ }^{1} \mathrm{H}_{-}{ }^{13} \mathrm{C}$ HMBC (solid arrows) spectra. A) Parts of the $1 \mathrm{D} 600 \mathrm{MHz}{ }^{1} \mathrm{H}-\mathrm{NMR}$ spectra are shown at the top and at the lefthand edge. B) Part of the $1 \mathrm{D} 600 \mathrm{MHz}{ }^{1} \mathrm{H}-\mathrm{NMR}$ spectrum is shown at the top edge, and the part of the $150 \mathrm{MHz}{ }^{13} \mathrm{C}-\mathrm{NMR}$ spectrum at the left-hand edge.

multiplicity, coupling constants and integrals in ${ }^{1} \mathrm{H}$ spectra are in agreement with expected structures. Because of two aromatic rings (in $\mathbf{1}$ one ring), the most signals (1-10) were found in the range 9.5-8.0 ppm in ${ }^{1} \mathrm{H}$ spectra and 190 $120 \mathrm{ppm}$ in ${ }^{13} \mathrm{C}$. The signals of $\mathrm{H}-7$ and $\mathrm{C}-7$ nuclei were found in the higher magnetic field $(\delta(\mathrm{H}-7)=6.7 \mathrm{ppm}, \delta(\mathrm{C}-7)$ $=66.5 \mathrm{ppm})$ as well as ${ }^{1} \mathrm{H}$ and ${ }^{13} \mathrm{C}$ nuclei signals of methyl (5; $\left.\delta\left(\underline{\mathrm{C}}_{3}\right)=2.44 \mathrm{ppm}, \delta\left(\underline{\mathrm{CH}}_{3}\right)=21.3 \mathrm{ppm}\right)$ and methoxy groups $\left(7,9 ; \delta\left(\mathrm{OC}_{3}\right) \approx 4.00 \mathrm{ppm}, \delta\left(\mathrm{O}_{\mathrm{C}} \mathrm{H}_{3}\right) \approx 56.00 \mathrm{ppm}\right)$. Cross signals obtained in $2 \mathrm{D}{ }^{1} \mathrm{H}-{ }^{1} \mathrm{H}$ COSY and ${ }^{1} \mathrm{H}-{ }^{13} \mathrm{C} \mathrm{HMBC}$ spectra are presented in Figure 2 together with the atom-numbering scheme used for the assignment of the NMR spectra. Coupling constants which confirmed presented structures are marked by dash and solid arrows in structure 10, and by red squares or ovals in spectra pictures (Figure $3 \mathrm{~A}, \mathrm{~B}$ ). By COSY spectrum we distinguished pairs from pyridine ring $(\mathrm{H}-2, \mathrm{H}-6 ; \mathrm{H}-3, \mathrm{H}-5)$ and those from phenyl moiety $(\mathrm{H}-10, \mathrm{H}-14 ; \mathrm{H}-11, \mathrm{H}-13)$. Substitutions of 2-bromoacetophenones on isonicotinamide unit were confirmed by $\mathrm{H}-7$ / C-2, C- 6 cross signal in ${ }^{1} \mathrm{H}-{ }^{13} \mathrm{C}$ HMBC spectra. Other signals marked in Figure $3 \mathrm{~B}$ confirmed the structures of synthesized molecules. 1D NMR spectra can be found in Supplementary files.

\section{Antifungal Activity}

Two different concentrations (10 and $100 \mu \mathrm{g} \mathrm{mL}-1$ ) of compounds (1-12) were evaluated for their antifungal activities in vitro against four fungal pathogens: $M$. phaseolina, S. sclerotiorum, F. oxysporum and F. culmorum (Table 3).

Inhibition of isonicotinamide derivatives on the $M$. phaseolina Among investigated compounds, five of them showed good inhibitory activity against M. phaseolina. More precisely, compound 10 at concentrations of 10 and $100 \mu \mathrm{gL}^{-1}$ showed the highest inhibitory activities. The compounds 7 


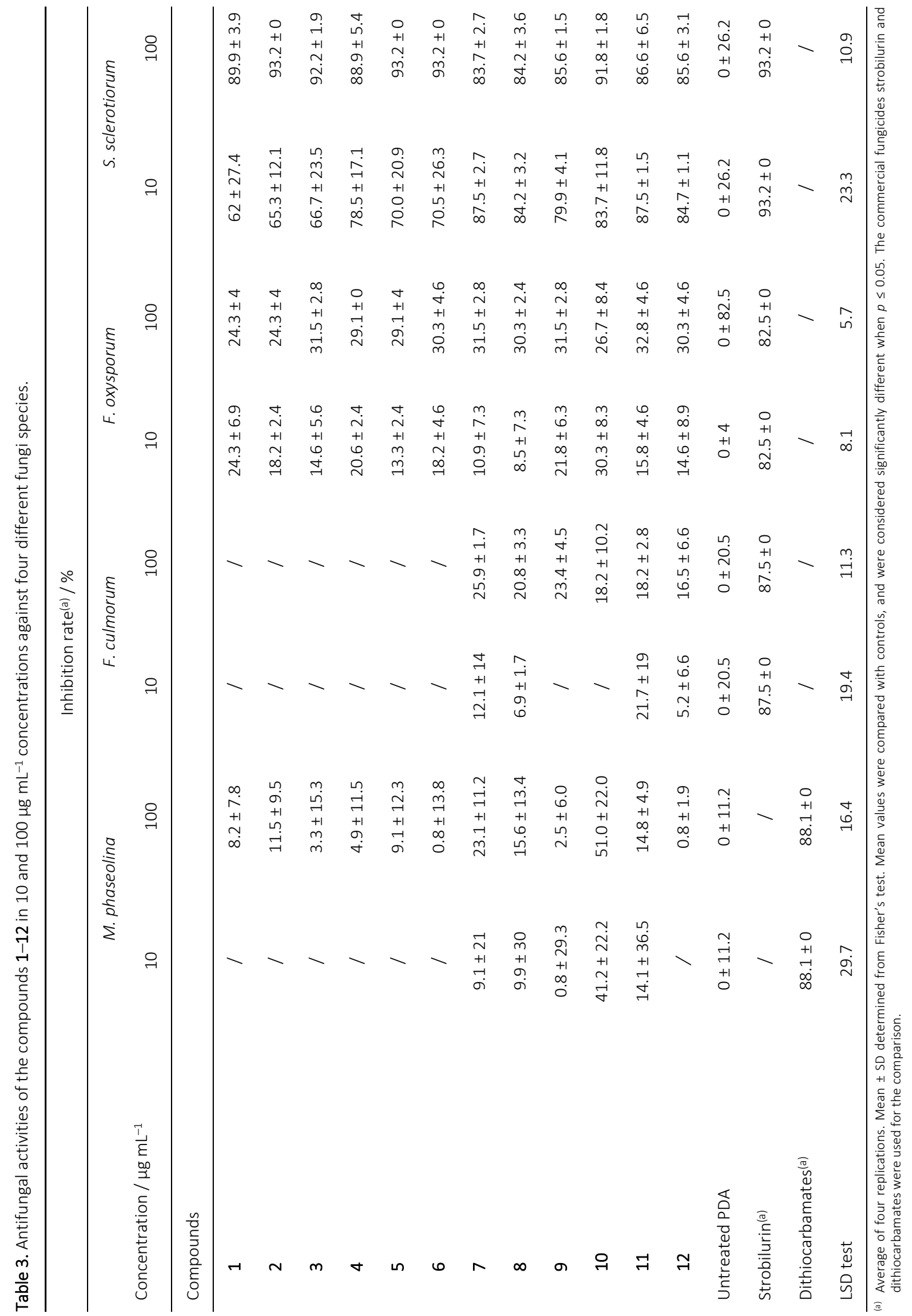

DOI: $10.5562 /$ cca3527 
and 8 at concentration of $100 \mu \mathrm{gL}^{-1}$ showed very good inhibitory activities. The compound $\mathbf{9}$ showed the lowest activity at concentration of $10 \mu \mathrm{g} \mathrm{mL}^{-1}$ against $M$. phaseolina, while at concentration of $100 \mu \mathrm{g} \mathrm{mL}^{-1}$ the lowest activity was observed for compounds $\mathbf{6}$ and $\mathbf{1 2}$. Compounds $\mathbf{1 - 6}$ at concentration of $10 \mu \mathrm{g} \mathrm{mL}^{-1}$ did not show any inhibitory activities against this fungus.

\section{Inhibition of isonicotinamide derivatives on the $F$. culmorum and $F$. oxysporum}

The compound $\mathbf{1 1}$ (at concentrations of 10 and $100 \mu \mathrm{gL}^{-1}$ ), as well as compounds $\mathbf{7 - 1 0}$ (at concentration of $100 \mu \mathrm{g} \mathrm{mL}^{-1}$ ) have shown inhibitory against $F$. culmorum without any significant statistical differences. The compounds 1-6 at both concentrations did not show any inhibitory activities against $F$. culmorum. Statistical significant differences were noted between untreated PDA and investigated compounds at concentration of $100 \mu \mathrm{g} \mathrm{mL}-1$.

Against $F$. oxysporum very good inhibitory activities have shown all compounds at concentration of $100 \mu \mathrm{gL}^{-1}$, while the compound $\mathbf{1 1}$ has shown the highest inhibitory effect. The compound $\mathbf{8}$ indicate the lowest activity against F. oxysporum. For all tested compounds at both concentrations statistical significant differences were indicated between them and untreated PDA.

Inhibition of isonicotinamide derivatives on the S. sclerotiorum All tested compounds have showed the highest inhibitory activity (83.7-93.2\%) against S. sclerotiorum in concentration of $100 \mu \mathrm{g} \mathrm{mL}^{-1}$, and all also showed strong inhibitory activities (62-87.5\%) in the concentration of $10 \mu \mathrm{gL}^{-1}$. The same trend of inhibitory activities is continued over the next $24 \mathrm{~h}$. The data are presented in the Table 3.

\section{CONCLUSIONS}

By rapid microwave irradiation ten novel isonicotinamide derivatives were prepared by the quaternization reactions of isonicotinamide with methyl iodide and nine differently substituted 2-bromoacetophenones. The microwave preparations were significantly faster with the yields up to 8 times, than the synthesis by conventional method. From the antifungal assay it was found that the most prepared compounds have moderate to weak activity against M. phaseolina and F. culmorum, while the inhibitory rate of F. oxysporum ranged $24.3-32.8 \%$ having statistically differences between control and tested compounds. All twelve isonicotinamide derivatives have a very high inhibitory rate against $S$. sclerotiorum, $62-87.5 \%$ in concentration of $10 \mu \mathrm{g} \mathrm{mL}^{-1}$ and $83.7-93 \%$ in concentration of $100 \mu \mathrm{gL}^{-1}$. Such findings are in accordance with previous studies of similar compounds, which also shown that pyridine carboxamide group displays considerable fungicidal activity.
Supplementary Information. Supporting information to the paper is attached to the electronic version of the article at: http://doi.org/10.5562/cca3527.

PDF files with attached documents are best viewed with Adobe Acrobat Reader which is free and can be downloaded from Adobe's web site.

\section{REFERENCES}

[1] R. Patel, A. Bhandari, AJADD, 2014, 2(1), 104-109.

[2] N. Tewatia, Abida, K. P. Namdeo, J. Chem. Pharm. Res. 2012, 4(3), 1794-1798.

[3] N. M. Khalifa, A. E. G. Amr, M. A. Al-Omar, E. S. Nossier, Russ. J. Gen. Chem. 2016, 86(12), 2785-2790. https://doi.org/10.1134/\$1070363216120409

[4] E. M. Sharshira, N. M. M. Hamada, Molecules 2011, 16(9), 7736-7745.

https://doi.org/10.3390/molecules16097736

[5] M. Kaya, E. Demir, H. Bekci, J. Enzyme Inhib. Med. Chem. 2013, 28(5), 885-893.

https://doi.org/10.3109/14756366.2012.692087

[6] Y. Shivaraj, M. H. Naveen, G. R. Vijayakumar, D. B. A. Kumar, J. Korean Chem. Soc. 2013, 57(2), 241-245. https://doi.org/10.5012/jkcs.2013.57.2.241

[7] J. J. Wang, K. C. Yao, S. S. Zhao, Y. H. Ye, Pestic. Biochem. Physiol. Articles in press, 2019.

[8] J. Sun, Y. Zhou, Molecules 2015, 20, 4383-4394. https://doi.org/10.3390/molecules20034383

[9] A. Patil, R. Jadhav, H. Raundal, L. Sharma, R. Badgujar, V. Bobade, J. Chem. Pharm. Res. 2014, 6(8), 218-223.

[10] Z. L. Ren, H. Liu, D. Jiao, H. T. Hu, W. Wang, J. X. Gong, A. L. Wang, H. Q. Cao, X. H. Lv, Drug Dev. Res. 2018, 79(6), 307-312. https://doi.org/10.1002/ddr.21469

[11] M. Faisal, M. I. Jihad, J. Global Pharma Technol. 2018, 10(03), 134-141.

[12] C. Pathak, R. R. Singh, S. Yadav, N. Kapoor, V. Raina, S. Gupta, A. Surolia, IUBMB Life. 2014, 66(3), 201211. https://doi.org/10.1002/iub.1252

[13] H. Bibi, H. Nadeem, M. Abbas, M. Arif1, BMC Chemistry 2019, 13:6.

https://doi.org/10.1186/s13065-019-0518-6

[14] L. Zhang, X. S. Deng, G. P. Meng, C. L. Zhang, C. CongChong, X. L. Zhao, H. Qing-Chun, C. Hu, Lett. Drug Des. Discov. 2018, 15(1), 70-83. https://doi.org/10.2174/1570180814666170929093258

[15] A. Mohammadi-Farani, A. Foroumadi, M. Rezvani Kashani, A. Aliabadi, IJBMS 2014, 17(7), 502-508.

[16] M. El-Naggar, H. Almahli, H. S. Ibrahim, W. M. Eldehna, H. A. Abdel-Aziz, Molecules 2018, 23, 1459. https://doi.org/10.3390/molecules23061459

[17] M. Peng, L. Shi, and S. Ke, Chem. Cent. J. 2017, 11:109. https://doi.org/10.1186/s13065-017-0338-5 
[18] W. M. Eldehna, G. S. Hassan, S. T. Al-Rashood, T. AlWarhi, A. E. Altyar, H. M. Alkahtani, J. Enzyme Inhib. Med. Chem. 2019, 34, 322-332.

https://doi.org/10.1080/14756366.2018.1547286

[19] S. Watanuki, K. Matsura, Y. Tomura, M. Okada, T. Okazaki, M. Ohta, S. Tsukamoto, Bioorg. Med. Chem. 2011, 19(18), 5628-5638.

https://doi.org/10.1016/j.bmc.2011.07.030

[20] S. Akhtar, Z. S. Saify, M. Arif, N. Mushtaq, A. Dar, A. Ahmed, Pak. J. Physiol. 2006, 2(1), 1-7.

[21] M. R. Khajouei, A. Mohammadi-Farani, A. Moradi, A. Aliabadi, Res. Pharm. Sci. 2018, 13(3), 262-272. https://doi.org/10.4103/1735-5362.228956

[22] M. J. Ahsan, M. Amir, RRJMC 2012, 1, 1-4.

[23] M. Ohba, T. Oka, T. Ando, S. Arahata, A. Ikegaya, H. Takagi, N. Ogo, K. Owada, F. Kawamori, Q. Wang, LJ. Saif, A. Asai, Chem. Pharm. Bull. 2016, 64(5), 465-475. https://doi.org/10.1248/cpb.c16-00001

[24] J. W. Dhore, G. B. Pethe, S. P. Wagh, G. D. Thorat, Arch. Appl. Sci. Res. 2011, 3, 407-414.

[25] J. Wu, S. Kang, L. Luo, Q. Shi, J. Ma, J. Yin, B. Song, D. $\mathrm{Hu}$, S. Yang, Chem. Central J. 2013, 7:64. https://doi.org/10.1186/1752-153X-7-64

[26] Z. Wu, D. Hu, J. Kuang, H. Cai, S. Wu, W. Xue, Molecules 2012, 17, 14205-14218. https://doi.org/10.3390/molecules171214205

[27] B. Kozlevčar, I. Leban, I. Turel, P. Segedin, M. Petrič, F. Pohleven, Polyhedron 1999, 18, 755-762. https://doi.org/10.1016/S0277-5387(98)00350-7

[28] T. Komori, S. Ujita, T. Inoue, Chem. Abstr. 2010, 154, 64642.

[29] G. Scalliet, J. Bowler, T. Luksch, L. Kirchhofer-Allan, D. Steinhauer, K. Ward, M. Niklaus, A. Verras, M. Csukai,
A. Daina, R. Fonné-Pfister, PLoS ON, 2012, 7, e35429. https://doi.org/10.1371/journal.pone.0035429

[30] N. G. Ravichandra, Agrochemicals in Plant Disease Management, Scientific publishers, 2018, Jodhpur, India, pp 556.

[31] Pesticide Properties DataBase, University of Hertfordshire, U.K., 26 June 2015, accessed 7 May 2019.

[32] T. A. Aire, Anat. Embryol. 2005, 210, 43-49. https://doi.org/10.1007/s00429-005-0001-0

[33] T. Siber, V. Bušić, D. Zobundžija, S. Roca, D. Vikić-Topić, K. Vrandečić, D. Gašo-Sokač, Molecules 2019, 24, 1001.

https://doi.org/10.3390/molecules24061001

[34] P. Euteneuer, H. Wagentristl, S. Steinkellner, C. Scheibreithner, J. G. Zaller, Appl. Soil Ecol. 2019, 138, 88-93. https://doi.org/10.1016/j.apsoil.2019.02.020

[35] D. Jurković, J. Ćosić, K. Vrandečić, Pseudofungi and fungi of cereals and arable crops. Faculty of Agrobiotechnical Sciences Osijek, 2016, pp. 288.

[36] S. Stojanović, Poljoprivredna fitopatologija. Srpsko biološko društvo Kragujevac, 2004, pp. 777.

[37] M. Aćimović, Sunflower diseases (Bolesti suncokreta, in Serbian). Institute of Field and Vegetable Crops, Novi Sad, Serbia, 1998, pp. 734.

[38] J. Ćosić, D. Jurković, Poljoprivreda, 2001, 7, 5-9.

[39] V. Bušić, H. Pavlović, S. Roca, D. Vikić-Topić, D. GašoSokač, Croat. Chem. Acta 2017, 90, 425-433. https://doi.org/10.5562/cca2937

[40] SAS / STAT. User's guide, version 9.2, SAS Institute Inc., Cary, NC, USA.

[41] C.O. Kappe, Angew. Chem. Int. Ed. 2004, 43, 62506284. https://doi.org/10.1002/anie.200400655 